\title{
Centro de Investigación y Asistencia en Tecnología y Diseño del Estado de Jalisco, A.C.*
}

\author{
Zaira Yunuen García Carvajal,, Hugo Espinosa-Andrews²
}

RESUMEN: El Centro de Investigación y Asistencia en Tecnología y Diseño del Estado de Jalisco, A.C. (CIATEJ) es un Centro Público perteneciente al Consejo Nacional de Ciencia y Tecnología (CONACyt). CIATEJ es un centro interdisciplinario que realiza actividades de investigación, desarrollo tecnológico e innovación, ofrece servicios tecnológicos y formación de recursos humanos de alto nivel. Hoy en día, un número cada vez mayor de nanomateriales son incluidos en productos de terapia avanzada, medicamentos, y alimentos. Muchos de estos productos se encuentran en etapas tempranas de investigación y desarrollo, y algunos otros ya son comercializados. Sin embargo, las reivindicaciones para su comercialización se pueden ver obstaculizadas por exigencias normativas cada vez más complejas. En este sentido, CIATEJ es un socio estratégico que cuenta con personal especializado que puede asesorar, generar y proteger conocimiento de vanguardia desarrollando productos, procesos y servicios para para impulsar el desarrollo en este sector.

PALABRAS CLAVE: Investigación, desarrollo tecnológico, innovación, nanoalimentos, nanomedicina.

ABSTRACT: The Center for Research and Applied Technology in Jalisco (spanish acronym: CIATEJ) is part of the National Council of Science and Technology (spanish acronym: CONACyt). CIATEJ is an interdisciplinary institute where scientific research, technological development and innovation, technological services and postgraduate research programs (including Master of Science and Doctor of Philosophy) are performed. Today, a growing number of nanomaterials and nanoproducts are included in advanced therapy products, medicines, nutraceuticals and foods. Many of them are in research and development early-stages and some of them are already on the market. However, the claims for marketing are hampered by as complex regulatory requirements. In this sense, CIATEJ is a strategic scientific and technological partner because it has highly specialized personal who can advise, generate and protect up-to-date knowledge to develop innovative products, processes and services give a boost to the progress of these sectors.

KEYWORDS: Research, technology development, innovation, nanofoods, nanomedicine.

Recibido: 19 de agosto de 2016. Aceptado: 5 de octubre de 2016.

* Se agradece a los doctores Inocencio Higuera Ciapara, Rodolfo Hernández Gutiérrez, Alba A. Vallejo Cardona, Maricela González Avila, Ma. de los Angeles Sánchez Contreras, Eristeo García Márquez y a la maestra Fátima G. Ordoñez de la Cruz, por su apoyo para la recopilación de la información.

1 Unidad de Biotecnología Médica y Farmacéutica. Centro de Investigación y Asistencia en Tecnología y Diseño del Estado de Jalisco. Av. Normalistas No. 800, Colinas de la Normal, CP. 44270, Guadalajara, Jalisco, México. Correspondencia: (zgarcia@ciatej.mx).

2 Unidad de Tecnología Alimentaria. Centro de Investigación y Asistencia en Tecnología y Diseño del Estado de Jalisco, Camino el Arenero 1227, El Bajío arenal, C.P.45019, Zapopan Jalisco, México. Correspondencia: (hespinosa@ciatej.mx). 
El Centro de Investigación y Asistencia en Tecnología y Diseño del Estado de Jalisco, CIATEJ A. C., fue fundado el 24 de agosto de 1976 en la ciudad de Guadalajara como una asociación civil sin fines de lucro, enfocada a la asistencia tecnológica de las industrias de la joyería, calzado y vestido de la región. En 1982 se establecieron las bases para orientar las investigaciones del CIATEJ hacia las necesidades de la industria agroalimentaria, química y farmacéutica del país. En 1992 se convierte en una institución paraestatal y en el año 2000 fue integrado a la red de centros públicos pertenecientes al Sistema de Centros Públicos de Investigación del Consejo Nacional de Ciencia y Tecnología (CONACyT). Desde 1996, el CIATEJ ofrece servicios de calibración de instrumentos en las magnitudes de masa, volumen, presión y temperatura, así como asesoría y capacitación en el área de Metrología. Actualmente, los servicios de calibración en las magnitudes de masa y volumen se encuentran acreditados bajo la norma NMXEC-025-IMNC-2000. En el año 2003 se iniciaron las actividades de vinculación con el sector farmacéutico veterinario a través de la firma de un contrato para el desarrollo de una vacuna para la prevención de la mastitis en el ganado lechero. En el 2006 se inauguró la Unidad de Investigación de Calidad del Aire, dentro del marco de la firma de un Convenio de Colaboración Científica, Tecnológica y Académica en materia de Medio Ambiente. En el 2009 se firmó un Convenio General de Colaboración con el Consejo Regulador del Tequila (CRT), con la finalidad de impulsar sólidamente a uno de los sectores en donde el CIATEJ ha generado una mayor dinámica en la formación de recursos humanos, generación de conocimiento, y transferencia de servicios tecnológicos para el sector. En el

FIGURA 1. Distribución geográfica de las Unidades del CIATEJ.

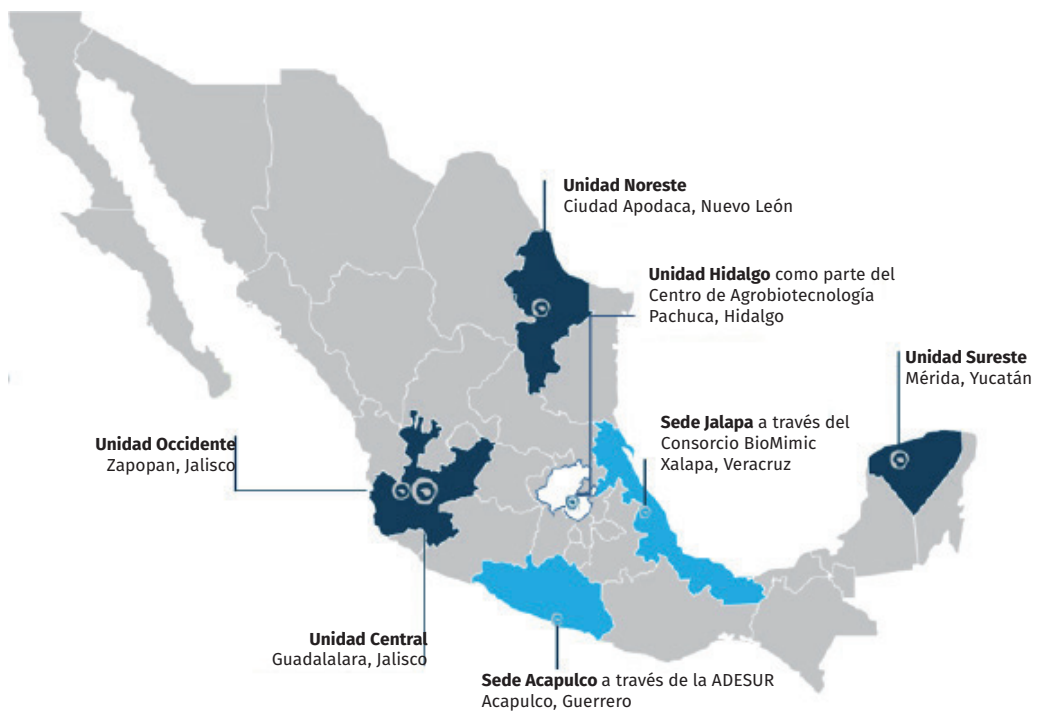


año 2013 el CIATEJ certificó su Oficina de Transferencia de Conocimiento con el Fondo Sectorial de Innovación Secretaría de Economía (FINNOVA).

En el 2016, la institución cumple 40 años impulsando el desarrollo tecnológico y transferencia de tecnología del país mediante la generación de conocimiento de vanguardia y la aplicación innovadora de la biotecnología. El CIATEJ cuenta con cuatro unidades de investigación en las ciudades de Guadalajara y Zapopan, Jalisco (Unidad Central y Zapopan, respectivamente); Mérida, Yucatán (Unidad Sureste) y Apodaca, Nuevo León (Unidad Noreste). Como parte de la estrategia de crecimiento institucional, el CIATEJ cuenta con un equipo de investigadores en la ciudad de Acapulco, Guerrero, en el proyecto regional denominado Alianza Estratégica para el Desarrollo Sustentable de la Región Pacífico Sur (ADESUR); y en la ciudad de Xalapa, Veracruz, en el consorcio denominado Bio-Mimic. En el centro del país el CIATEJ tendrá presencia dentro del marco del proyecto "Ciudad del Conocimiento", al formar parte del Centro de Agrobiotecnología en la ciudad de Pachuca, Hidalgo, en conjunto con el Centro de Investigación en Alimentación y Desarrollo, A.C. (CIAD).

\section{Líneas de investigación}

Las líneas de investigación, desarrollo tecnológico e innovación $(\mathrm{I}+\mathrm{D}+\mathrm{i})$ y servicios tecnológicos, servicios analíticos y metrológicos, gestión de posgrados y estudiantes que ofrece el CIATEJ son:

1. Biotecnología industrial

2. Biotecnología médica y farmacéutica

3. Biotecnología vegetal

4. Tecnología alimentaria

5. Tecnología ambiental

6. USAM: Laboratorios de Servicios Analíticos y Metrológicos

El CIATEJ cuenta con una matrícula de 147 estudiantes de posgrado inscritos en 5 programas de posgrado registrados en el Padrón Nacional de Posgrados de Calidad (PNPC) de la SEP-CONACyT:

1. Maestría en ciencia y tecnología del Posgrado Interinstitucional en Ciencia y Tecnología (PICyT),

2. Doctorado en ciencia y tecnología del PICyT,

3. Maestría en ciencias de la floricultura.

4. Maestría en ciencias en innovación biotecnológica (reciente creación).

5. Doctorado en ciencias en innovación biotecnológica (reciente creación).

Estos dos últimos presentan las opciones terminales en biotecnología médica farmacéutica, biotecnología agroalimentaria y ciencias ambientales. La 
característica principal de los posgrados del CIATEJ es la formación de capital intelectual de alto nivel científico y tecnológico, capaz de generar, innovar, aplicar y transmitir conocimientos actuales de vanguardia y socialmente relevantes, impactando en el sector productivo del país.

\section{Investigación en el campo de la nanotecnología}

Las investigaciones y desarrollos tecnológicos están orientados dentro de los temas prioritarios del PECiTI 2014-2018 en las áreas de desarrollo tecnológico: desarrollo de nanomateriales y de nanotecnología, y, salud: medicina preventiva y atención de la salud; y al interior del CIATEJ se desarrollan en las áreas de tecnología alimentaria y biotecnología médica y farmacéutica.

\section{Tecnología alimentaria}

El área de tecnología alimentaria atiende las necesidades de investigación y desarrollo tecnológico de la industria y de servicios, en el que se incluyen tanto a las empresas que elaboran alimentos y bebidas como otras industrias y servicios afines. Las aplicaciones de las nanociencias y nanotecnología en los alimentos es un campo relativamente nuevo, que muestra un crecimiento exponencial en los campos de la ciencia, la tecnología y los desarrollos comerciales. El CIATEJ es considerado pionero en el estado de Jalisco en el desarrollo tecnológico en el área de la nanotecnología aplicada al campo de la alimentación (www.jalisco.gob.mx/es/prensa/noticias/9521). Se espera que el desarrollo de aplicaciones, productos y procesos tecnológicos relacionados con la nanotecnología traerá múltiples beneficios en el sector de la alimentación, como la mejora de textura en los alimentos, el incremento de las sensaciones organolépticas, incremento en la biodisponibilidad de nutrientes, y el mejoramiento de empaques entre otros.

Los investigadores relacionados con esta área de investigación tienen presencia en las cuatro Unidades del CIATEJ agrupados en la línea de investigación de "Desarrollo y calidad de alimentos y bebidas". Las investigaciones desarrolladas en esta línea de investigación relacionada con las nanociencias y nanotecnologías abordan estudios fundamentales de ciencia-básica y ciencia-aplicada con la finalidad de obtener y caracterizar sustancias bioactivas y biomateriales; diseñar y validar las propiedades fisicoquímicas, funcionales y tecnológicas de las nanopartículas. Las sub-líneas de investigación en esta área están orientadas a comprender cómo las características fisicoquímicas de los nanomateriales pueden cambiar la estructura, textura, estabilidad y propiedades sensoriales de los alimentos (color, sabor, olor, consistencia, entre otras). La adquisición de infraestructura en el campo de la nanotecnología posiciona al área de tecnología alimentaria como un aliado estratégico para desarrollar proyectos de investigación básica y aplicada en esta materia. Por ejemplo, en el CIATEJ se cuenta con diferentes tecnologías 
para separación de sustancias bioactivas mediante tecnologías de vanguardia, entre las que podemos mencionar la extracción con fluidos súpercríticos, destilador molecular, tecnología de membranas cromatografía a contracorriente de alta resolución (HPCCC, por sus siglas en inglés), así como la identificación de las mismas mediante cromatografía de capa fina (TLC, por sus siglas en inglés), cromatografía de gases, cromatografía de líquidos de alta presión entre otros.

Contamos con tecnología de vanguardia en proyectos de I+D+i relacionados con la generación de nanopartículas y nanocápsulas empleando la tecnología de secador por aspersión a escala nano (figura 2), la cual permite obtener partículas en polvo de 1 a 2 órdenes de magnitud menores a los equipos convencionales de secado por aspersión; o en proyectos de encapsulación de principios bioactivos de baja solubilidad mediante la optimización de nanoemulsiones y nanosuspensiones (figura 3 ).

FIGURA 2. Nanosecador por aspersión B-90 (Büchi, Suiza).
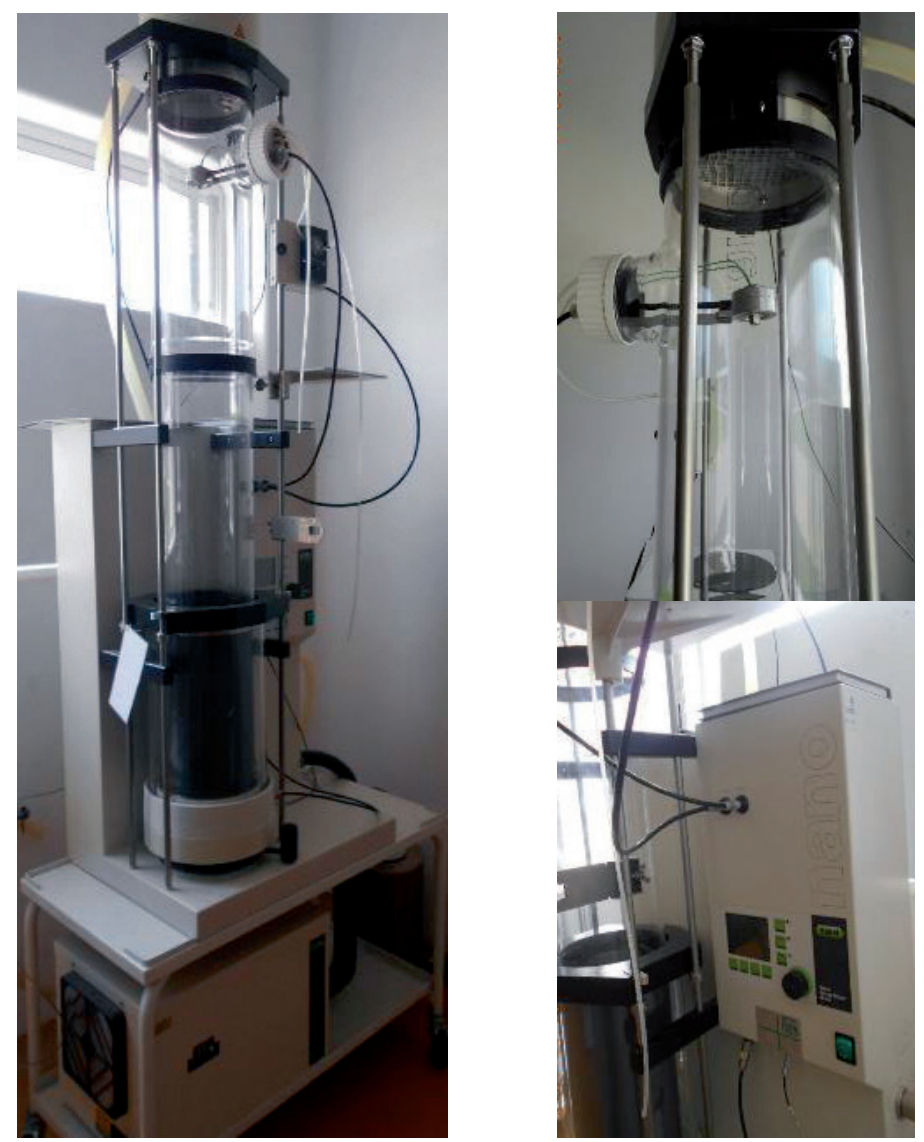
FIGURA 3. Homogeneizador de alta presión (Microfluidics, Estados Unidos).

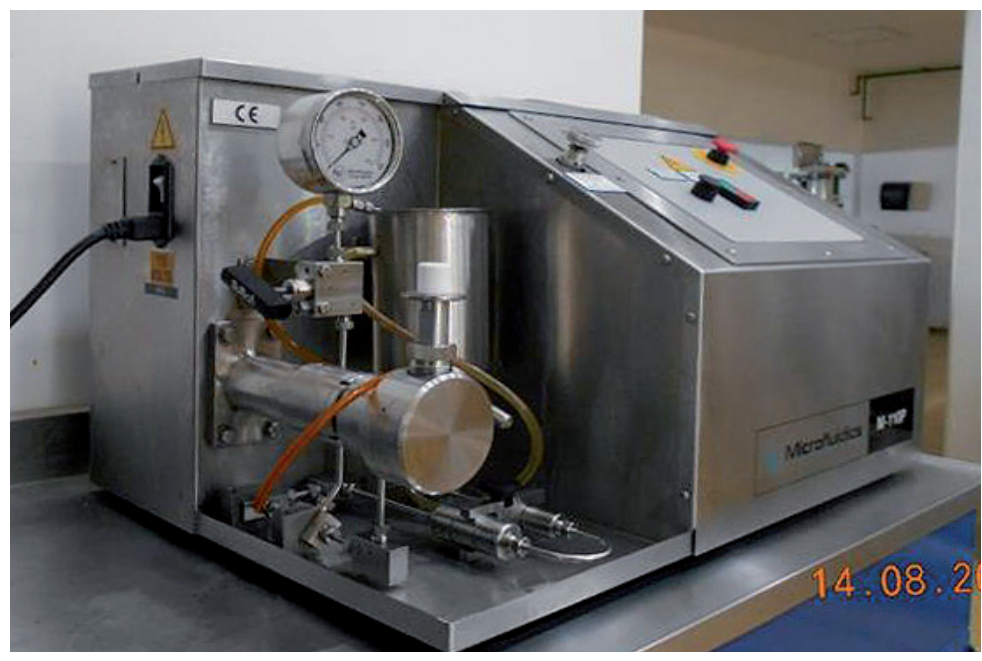

Estas tecnologías son aplicadas en el campo de la nanoencapsulación de aditivos, nutracéuticos, alimentos funcionales, aromas, proteínas, péptidos, entre otros. Entre las principales ventajas que ofrecen incluyen: 1) reducir la dosificación e incrementar el valor agregado de los productos bioactivos; 2) incrementar la biodisponibilidad de los principios activos; 3) mejorar la solubilidad y dispersabilidad de los principios bioactivos; 4) reducir los costos de I + D, y, 5) trabajar con volúmenes pequeños del principio activo. En general, la reducción de tamaño y distribución de las partículas incrementa el área superficial del principio activo, lo que repercute en un incremento en la velocidad de disolución aumentando la tasa de transferencia. En el campo de los alimentos, la distribución de tamaño de las partículas puede oscilar entre $10 \mathrm{~nm}-1 \mu \mathrm{m}$. Las aplicaciones de la nanotecnología aún son limitadas en el sector de alimentos, pero muestran un incremento importante en el área suplementos alimenticios y farmacéutica. En esta sublínea de investigación las investigaciones giran alrededor de:

1. Nanoencapsulación de aromas y sabores.

2. Desarrollo y validación de la funcionalidad de nanopartículas de sustancias bioactivas.

3. Diseño y funcionalización de principios bioactivos lipofílicos a partir de nanoemulsiones/nanosuspensiones.

\section{Biotecnología médica y farmacéutica}

La Unidad de Biotecnología Médica y Farmacéutica del CIATEJ surge como una alternativa para atender las demandas del sector salud humana y animal 
FIGURA 4. Simulador del tracto digestivo humano (Mx/a/2012/005418; por sus siglas en inglés: ARIS, Automatic and Robotics Intestinal System).

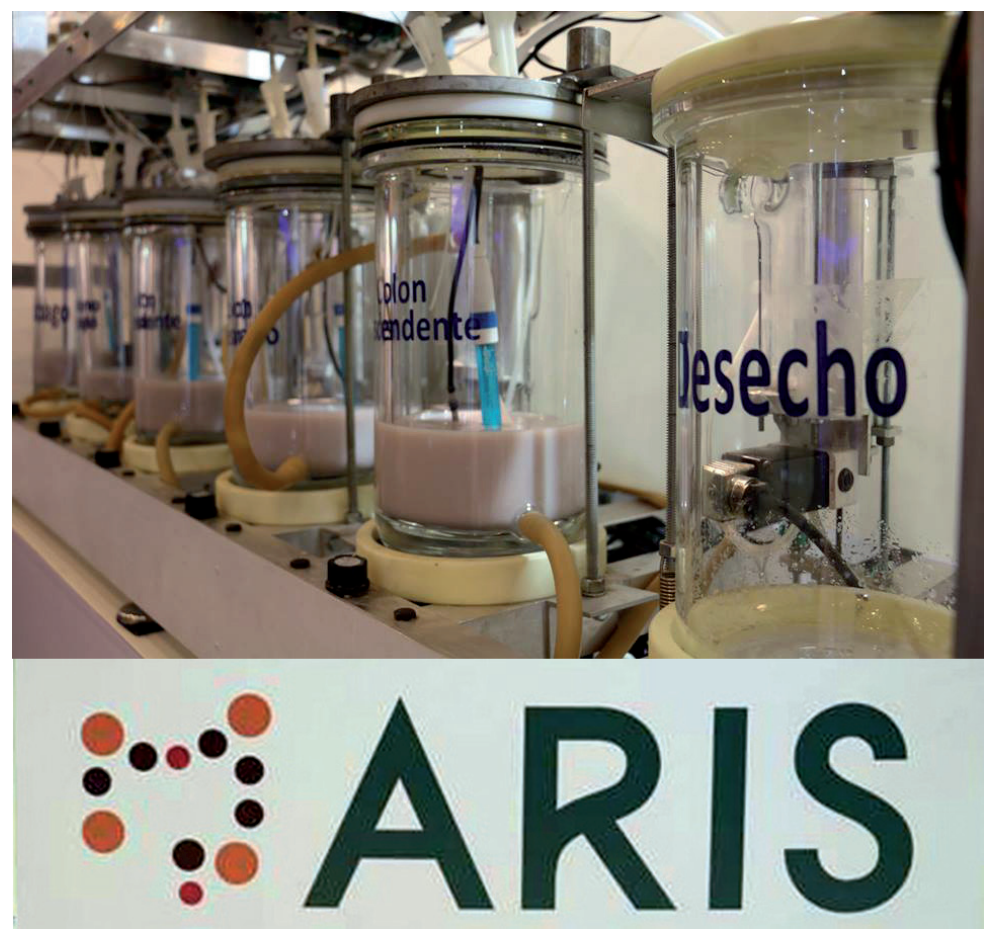

de nuestro país, a través del desarrollo de medidas profilácticas y/o terapéuticas, que favorezcan el crecimiento de ambos sectores. Está unidad cuenta con 18 investigadores y 3 cátedras CONACyT en las siguientes líneas de investigación: 1) desarrollo y evaluación de vacunas y compuestos inmunomoduladores; 2) desarrollo y validación de pruebas de diagnóstico molecular; y 3) desarrollo y evaluación de productos con potencial terapéutico.

Los investigadores de esta unidad que trabajan con nanotecnología se orientan en investigaciones relacionadas con:

\section{Tecnología farmacéutica de formas de administración vía oral}

La industria farmacéutica está experimentando ciertas transformaciones, como la evolución de medicamentos tradicionales a medicamentos especializados dirigidos a grupos específicos. Los enfoques de sistemas de entrega de fármacos actuales deben ser rediseñados, mejorados y optimizados para lograr la individualización. Esto obliga al desarrollo de nuevas e innovadoras formas de dosificación mediante la aplicación de la nanotecnología, lo que podría superar las limitaciones de las formulaciones farmacéuticas actuales. Sin 
embargo, aún existen retos que superar y nuevas necesidades no satisfechas en el mercado para proponer soluciones funcionales en la administración especializada de fármacos por la vía oral. Las actividades científicas de CIATEJ en este sentido se enfocan en:

1. Diseñar, mejorar y optimizar formas de dosificación oral con versatilidad de encapsulamiento y de liberación inteligente, tomando en cuenta las propiedades fisicoquímicas de los materiales de partida que aseguren que los ingredientes encapsulados son liberados en el momento y lugar adecuado del tracto gastrointestinal, incrementando la posibilidad de acción de los ingredientes contenidos.

2. Evaluar la funcionalidad de las formas de dosificación oral en términos de: 1) digestibilidad del prototipo; 2) determinación del perfil de liberación del activo, 3) impacto de la formulación (sin ingrediente activo) sobre la microbiota intestinal empleando un simulador del tracto digestivo humano (figura 4).

3. Acompañamiento científico y tecnológico para el desarrollo de nanoproductos innovadores en fases de I+D+i.

\section{Evaluación de toxicidad in vitro de nanoproductos}

La industria agrícola y agroalimentaria de nuestro país exporta productos a nivel global y de manera cotidiana; esto involucra el cumplimiento de regulaciones sanitarias. Innovaciones en este sector se han enfocado al desarrollo de: 1) nanocápsulas conteniendo nutrientes (vitaminas, aceites, etc.); 2) nanosensores embebidos en el envasado de alimentos, los cuales advierten de contaminación química o bacteriana; 3) adición de nanoestructuras en la superficie de los paquetes que podrían ayudar a reducir/minimizar la carga bacteriana en contacto con el alimento extendiendo su vida de anaquel.

Sin embargo, los productos alimentarios no están sujetos a regulaciones estrictas en materia de nanotecnología. Por lo general, los fabricantes no tienen que declarar que han utilizado nanotecnología, o que su alimento contiene formulaciones de tamaño nanométrico. Aunado a esto, los consumidores en todo el mundo son cada vez más conscientes de lo que consumen, lo que ocasiona que se planteen preguntas sobre el origen y seguridad de los nanoproductos de consumo alimentario. A pesar de los grandes esfuerzos y logros hechos en materia de nanotecnología en México, nuestro país no cuenta con lineamientos sobre la seguridad alimentaria de los nanoproductos.

En CIATEJ estos problemas pueden ser abordados mediante la evaluación y seguimiento de nanomateriales presentes en productos nutracéuticos o farmacéuticos empleando un sistema de digestión ex vivo (ARIS), el cual está adaptado con microbiota intestinal de población mexicana sana. Este sistema es una herramienta útil e innovadora para evaluar la funcionalidad de productos funcionales del sector agroalimentario y farmacéutico del país. 
Los esfuerzos de esta sublínea de investigación están orientados en establecer las metodologías y técnicas apropiadas que permitan evaluar la funcionalidad en términos de seguridad y eficacia de nanoproductos.

También en la Unidad de Biotecnología Médica y Farmacéutica se han desarrollado investigaciones científicas relacionadas con la nanotecnología en terapia avanzada:

\section{Medicamentos de terapia avanzada}

Los medicamentos de terapia avanzada son una categoría de productos innovadores y comprenden medicamentos de terapia génica, terapia celular somática y productos de ingeniería tisular. La mayoría de estos productos se encuentran en las fases de I+D+i, impulsados por académicos, empresas de base tecnológica, y organizaciones sin fines de lucro. La complejidad de la clasificación de productos, procedimientos onerosos para las combinaciones de medicamentos de terapia avanzada de dispositivos médicos, las incertidumbres que rodean los procedimientos de certificación, la falta de armonización de importación - exportación, las normas y procedimientos divergentes para aspectos críticos del desarrollo en los diferentes países-. Actualmente, la nanotecnología desempeña un papel importante en la medicina regenerativa y en el desarrollo de órganos artificiales. Los avances se han dirigido a comprender las interacciones entre los dispositivos médicos y las células; desarrollo de nanomateriales inteligentes, nanopartículas fluorescentes como puntos cuánticos, nanopartículas magnéticas, nanotubos de carbono, grafeno, entre otros. Su finalidad es dar seguimiento a células y mejorar las propiedades de los implantes como el cartílago de pabellón auricular, epitelio gastrointestinal, hueso cortical, tendón y ligamento.

CIATEJ también ha incursionado en emplear la nanotecnología para la detección de cáncer. Varios estudios han demostrado que el suero de pacientes con cáncer contiene anticuerpos que reaccionan con un grupo de autoantígenos asociados con tumores denominados (TAA). En este sentido en el CIATEJ se busca desarrollar dispositivos para detectar diferentes tipos de líneas celulares de cáncer a base de proteínas y nanopartículas de oro. La detección de glicoproteínas como biomarcadores potenciales para el cáncer de mama en etapas tempranas (I y II) de la población mexicana es de vital importancia para su prevención dado que no se manifiestan síntomas que indiquen la presencia de esta enfermedad. La adquisición de infraestructura básica y la experiencia de sus investigadores en el campo de la nanotecnología posicionan al área de biotecnología farmacéutica como un aliado estratégico para desarrollar proyectos de investigación básica y aplicada en esta materia. Por ejemplo, en proyectos de I+D+i relacionados con la generación de andamios poliméricos conteniendo nanopartículas y nanocápsulas han sido fabricados empleando técnicas de electrohilado y de bioimpresión 3D (figura 5). 
FIGURA 5. Bioimpresora 3D con aditamentos para la impresión de geles poliméricos y estructuras nanométricas contenidas en los geles.

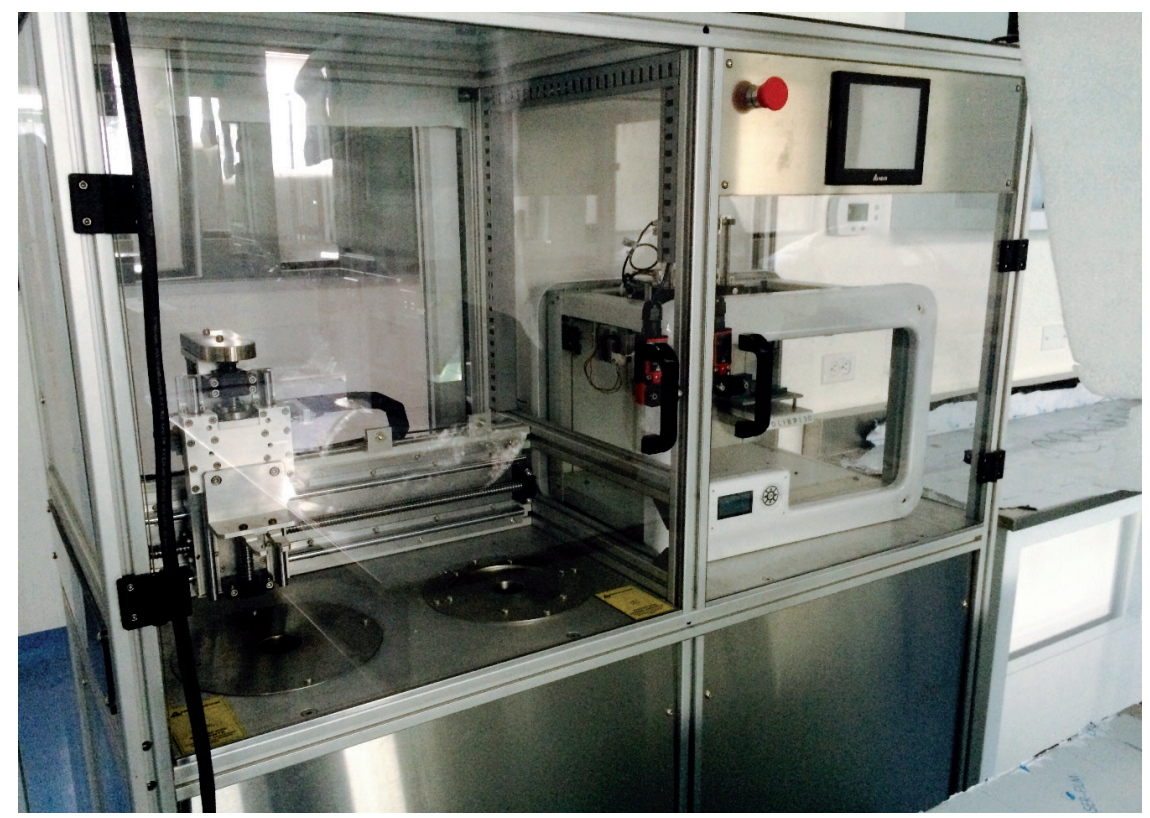

Las actividades científicas de CIATEJ en este sentido son:

1. Diseñar, mejorar y optimizar biomateriales de modo que sirvan como andamios para aplicaciones en ingeniería tisular incluyendo o no nanomateriales.

2. Diseñar, mejorar, optimizar, estandarizar, validar metodologías y técnicas de acuerdo con normativa armonizada (ISO 10993) a medida que sean de utilidad para la evaluación de la seguridad y eficacia de dispositivos médicos y por ende en el registro sanitario.

3. Desarrollo y evaluación de nanoproductos con potencial terapéutico.

4. Acompañamiento científico para el desarrollo de productos innovadores en fases de $\mathrm{I}+\mathrm{D}+\mathrm{i}$, mejora y optimización de los ya existentes.

\section{Proyectos CONACyT}

Algunos de los proyectos recientemente aprobados en las diferentes convocatorias de CONACyT relacionados con las NyN son:

1. Interacciones moleculares en sistemas nanoencapsulados. Responsable técnico: Dr. Inocencio Higuera Ciapara. 
2. Desarrollo de hidrogeles para reconstrucción auricular de pacientes quemados.

Responsable técnico: Dra. Zaira García Carvajal.

3. Funcionalización de nanopartículas de oro y magnéticas para detección y tratamiento de células cancerosas segunda Etapa.

Responsable técnico: Dr. Rodolfo Hernández Gutiérrez.

4. Nanoestructuras autoensambladas a base de biopolímeros naturales y su evaluación estabilizante de hierro para atender la desnutrición infantil.

Responsable técnico: Dr. Jorge Alberto García Fajardo.

5. Estudio de nanocomplejos como matrices de liberación controlada de compuestos bioactivos.

Responsable técnico: Dr. Hugo Espinosa Andrews.

Algunos de estos proyectos se desarrollan en colaboración con instituciones como el Instituto Nacional de Rehabilitación "Luis Guillermo Ibarra Ibarra”, la Universidad de Guadalajara (CuTonalá, CUCIENEGEA, CUCEI), la Universidad Michoacana de San Nicolás de Hidalgo, Universidad Veracruzana, Universidad Autónoma del Estado de México, Universidad Autónoma Metropolitana, el Centro de Nanociencias y Nanotecnología de la UNAM, el CIVESTAV y la Universidad de Münster, Alemania (Convenio firmado y vigente); y con empresas de base tecnológica como InMateriis, S.A. de C.V. e InterLatinColibri.

\section{Redes CONACyT}

Desde el año 2014 el CIATEJ pertenece a la Red Nacional de Investigación, Innovación y Desarrollo Tecnológico en Alimentos Funcionales y Nutracéuticos (www.alfanutra.com). Esta red interinstitucional tiene como objetivo conjugar el conocimiento integral, multidisciplinario, y las capacidades con las que cuenta la red de centros públicos de investigación del CONACyT, que se requiere para la obtención exitosa de ingredientes funcionales, desarrollo y diseño de alimentos funcionales y nutracéuticos, que generen conocimiento y riqueza.

\section{SINANOTOX. Red Temática de Nanociencias y Nanotecnología}

Recientemente, el CIATEJ se ha incorporado a la Red Temática de Nanociencias y Nanotecnología del CONACyT dentro de la iniciativa denominada "Sistema Nacional de Evaluación Nanotoxicológica SINANOTOX". Las actividades científicas del CIATEJ se enfocarán en:

1. Diseñar, estandarizar y validar las metodologías y técnicas que permitan estudiar la digestibilidad, disolución, y degradación de 
nanoproductos y nanoalimentos empleando el sistema de digestión ex vivo.

2. Acompañamiento científico para el desarrollo de productos innovadores en fases de I+D+i, así como la mejora y optimización de los ya existentes.

\section{Perspectivas a 5 años}

La adopción de nuevas tecnologías como lo son la nanotecnología, biotecnología y biomedicina aplicadas a productos de consumo humano deben enfrentar varios retos científicos, tecnológicos, de legislación, y la aceptación por los consumidores, entre otros. Ejemplos de estos retos son: 1) cumplimiento de una serie de requisitos sanitarios debidamente justificados para su salida al mercado, 2) cumplimiento de un exigente control de calidad, 3) cumplimiento de buenas prácticas de fabricación, por mencionar algunos.

A pesar de esto, muchas de las innovaciones en productos para consumo humano aplican la nanotecnología, dado que están proporcionando soluciones únicas con lo que aseguran el éxito comercial. A pesar del impacto de estos avances en la condición de la salud humana y alimentación, muchos de los estudios en humanos aún son marginales, y se espera que para la próxima década este tipo de estudios aumente considerablemente.

Por lo tanto, el mirar hacia adelante y visualizar las tendencias en nanotecnología con aplicaciones innovadoras para la salud y alimentos es un reto para CIATEJ. Por lo que nuestras perspectivas de trabajo se enfocan en:

1. Desarrollo de nanopartículas para proteger compuestos de interés contra el medio ambiente maximizando sus propiedades organolépticas y funcionales, minimizando su dosificación.

2. Desarrollo de nanopartículas que permitan una dosificación inteligente y controlada de los principios bioactivos.

3. $\mathrm{I}+\mathrm{D}+\mathrm{i}$ en tecnologías para la producción de nanomateriales para la salud

4. I+D+i en tecnologías, metodologías, productos para cuestiones de seguridad y salud relativas a los nanomateriales.

5. Educación en nanotecnología para la población y formación de recursos humanos en esta área.

6. Acompañamiento científico para las empresas interesadas en invertir en soluciones innovadoras para la salud y alimentos.

7. Desarrollo de nanoproductos innovadores que sean susceptibles de propiedad intelectual para su transferencia y licenciamiento al sector productivo.

8. Consolidación de línea de investigación de investigación e innovación en nanopartículas y nanoemulsiones como matrices de liberación controlada de compuestos bioactivos. 
9. Consolidación de la línea de investigación "Diseño y evaluación de herramientas biotecnológicas enfocadas a medicina regenerativa" en CIATEJ y socialización de la misma al sector productivo.

10. Incursionar en la aplicación innovadora de las nanociencias y la nanotecnología en las diferentes áreas del CIATEJ: biotecnología industrial; biotecnología ambiental y biotecnología vegetal.

\section{Espacios web institucionales}

- <www.ciatej.mx>.

- $\quad<$ http://www.picyt.edu.mx/>. 